\title{
Research on the Reliability of Electrical Automation Control Equipment Based on Servo Motor
}

\author{
Runze Gao \\ Harbin Institute of Technology, Harbin, Heilongjiang, China
}

Keywords: Servo motor, Electrical automation control, Reliability study

\begin{abstract}
With the continuous development of our society, various science and technology are constantly innovating. In recent years, electrical automation technology has been continuously promoted and used. The reasonable use of electrical automation control technology can improve its stability and performance in specific work. Reliability also provides technical support for various production activities. Therefore, the research in this article starts with the significance of improving the reliability of electrical automation control equipment, discusses specific measures to improve the reliability of electrical automation control equipment, and hopes to provide relevant people with reference opinions.
\end{abstract}

\section{Introduction}

At this stage, the application of electrical automation has become very popular in society, achieving the goal that electrical equipment can operate on its own, and with the rapid development of scientific information technology, the application of electrical automation in related industries is very popular. Because electrical automation control is of great help to promote China's social and economic development, electrical automation control technology has gradually become a standard for testing the development of China's electronics and other industries, and has attracted the attention of many professionals. In addition, electrical automation control technology also has the advantages of improving working conditions, improving the quality and efficiency of work, and enhancing economic benefits. However, at this stage, people are no longer limited to the demand for power supply. The stability of the electrical automation equipment during operation is more important, because the reliability of the control equipment can be guaranteed by improving the stability.

\section{Status Analysis of Reliability of Electrical Automation Control Equipment}

The working environment in different working places is also very different. Some working environments will feel very relaxed when working, but some working environments are very poor. The environment of electrical and automation equipment during work will be very large. Changes, the changes produced by these environments will have a negative effect on the equipment, and it will also bring difficulties to the staff. These factors are mainly reflected in the three aspects of electromagnetic interference, mechanical effects and climatic factors. Control, but the remaining two factors can be controlled, and relevant personnel need to pay more attention to this aspect.

Because the components required for electrical equipment are manufactured by different manufacturers, this also results in great differences in the quality of these components. Substandard quality components reduce the reliability of the control equipment. At this stage, the competition in the market is becoming increasingly fierce, and some component manufacturers will engage in unfair competition, ignoring the quality of products. In this case, the manufacturer only pays attention to the price, but does not pay attention to the quality of the components, which also reduces the reliability of the control equipment, which is not conducive to its normal work. Generally speaking, the component manufacturing enterprises are not large, and there are very few types of components that can be guided. Without an effective quality supervision department, the quality of components cannot be guaranteed. Because there are many companies that produce 
components, the price of components is relatively low, causing electrical and automation equipment manufacturers to ignore the quality aspect when purchasing, which will control the equipment to become very unreliable at work, which is harmful to electrical automation. The life of the equipment can also have a big impact.

Because the working environment is very complicated and the number of automation equipment is relatively large, it will be difficult to operate. This requires the ability of the operator to meet the standards. If the operator has unreasonable behavior during operation, it will seriously affect To the performance of the equipment; in addition, the maintenance of the equipment has also caused greater difficulties for the staff, the staff must pay more attention to these aspects in order to improve the reliability of electrical automation control equipment.

\section{Factors Affecting the Reliability of Control Equipment for Electrical Automation}

With the continuous development of China's society and economy, the popularity and application of electrical and automation equipment have also caused more and more manufacturers to start producing equipment components, including some smaller manufacturers. In order to save production costs, they want to use Low prices to attract buyers, the lack of strict production systems and standards in the specific component production process, resulting in many equipment components are unqualified and of poor quality. If unqualified components are used in the electrical automation production process, it will inevitably affect the entire production, cause the electrical automation equipment to fail to work properly, and bring economic losses to the enterprise. In serious cases, it will also lead to safety accidents. The occurrence of injuries and deaths can be said to be very harmful.

In addition to the control equipment reliability that affects electrical automation, the poor quality of the equipment components mentioned above may also be caused by environmental factors. Although the current electrical automation control equipment is widely used, it will be affected by environmental factors in the specific work, the temperature level and the pressure value will affect the performance of the equipment. In addition, electromagnetic will also affect the reliability of electrical automation equipment. In severe cases, it will also cause safety accidents and cause loss of people's lives and property. Therefore, relevant personnel must pay more attention to environmental issues. There are irregularities in the operation of the relevant staff of the enterprise during the operation of the equipment, which will also adversely affect the reliability of the electrical automation control equipment. At present, some operators of electrical automation control equipment the performance and structural characteristics are not well understood, and the equipment may be damaged due to operating errors in specific work. In addition, many companies do not pay much attention to the maintenance and maintenance of electrical and automation equipment. In this case, the equipment is prone to failure. Once the equipment fails, the equipment will not work properly and will cause certain losses to the enterprise.

There are irregularities in the operation of the relevant staff of the enterprise during the operation of the equipment, which will also adversely affect the reliability of the electrical automation control equipment. At present, some operators of electrical automation control equipment the performance and structural characteristics are not well understood, and the equipment may be damaged due to operating errors in specific work. In addition, many companies do not pay much attention to the maintenance and maintenance of electrical and automation equipment. In this case, the equipment is prone to failure. Once the equipment fails, the equipment will not work properly and will cause certain losses to the enterprise.

\section{Specific Measures to Improve the Reliability of Electrical Automation Control Equipment}

If you want to improve the reliability of electrical automation equipment, you must first formulate a scientific and reasonable design scheme. In the specific scheme design, you must first understand the performance and characteristics of the equipment and master its related parameters. Second, the specific application conditions of the equipment, the type of equipment construction 
and the size of the equipment must be understood and mastered. In the specific scheme design work, it is necessary to reasonably select the components and materials used to control the production costs while ensuring the quality as much as possible. Finally, the design plan formulated must be confirmed repeatedly to increase the science and rationality of its design.

For electrical automation control equipment, the selection of components is very important. First of all, we must understand the qualifications of component manufacturers, do market research in advance, and the quality of purchased components must meet standards. In addition, the components have very high requirements for the working environment and are easily affected by temperature and humidity. Therefore, the selected components must also have a good heat dissipation function. Finally, the inspection and testing of components must be strengthened, and components that do not meet the standards must not be used. It should also be noted that during the subsequent use of the equipment, the working conditions of different components can be recorded and the data can be archived. In the future, when selecting components, it can be used as a reference basis. Select as appropriate and quality as possible as possible. This is an important way to improve the reliability of the entire equipment. If you want to improve the reliability of electrical automation control equipment, you must also strengthen the climate protection measures of electrical automation control equipment, because temperature and humidity will also affect the reliability of electrical automation equipment. China has four distinct seasons, especially in winter, when the humidity is low and the humidity is relatively large. This can easily cause the water on the automation equipment to freeze together. In such a long-term humid environment, the equipment is very useful for erosion and Corrosion affects the service life of the equipment. Therefore, it is necessary for relevant companies to strengthen their attention, and to take relevant protective measures for electrical automation equipment. In actual work, sealing and potting can be used to isolate the equipment from the external environment, reduce the temperature and humidity Impact on it and prolong the service life of the equipment.

Want to improve the reliability of electrical automation control equipment In addition to the measures we mentioned above, it is also very important to improve the basic quality of the operator. The operator of the equipment is the main operator and plays a pivotal role. Relevant enterprises should change the traditional recruitment mode and improve the recruitment conditions. They can establish cooperative relations with universities, hire college graduates with professional quality standards, and do pre-job training. To hire professionals to specifically explain the use instructions and operating specifications of the enterprise equipment, the enterprise should also establish a corresponding assessment mechanism. For those who have not passed the assessment, they must be retrained before they can enter the job post. Finally, in order not to affect the normal use of the equipment, it is necessary to do a good job of equipment maintenance and repair, and to standardize the management of automated equipment. You can also use modern information technology on a large scale to constantly monitor the operating status of the automation equipment, and resolve any abnormalities as soon as possible. At the current stage, most Chinese enterprises only focus on the use of electrical automation control equipment, but do not pay enough attention to the safety and reliability of electrical automation control equipment, which will adversely affect the work of electrical control equipment. This shows that enterprises need to change their own ideas and realize more scientific and reliable equipment design. First of all, companies must change their thinking, clarify the role played by the reliability of control equipment, increase the emphasis on equipment maintenance, and conduct research on the emergence of control equipment to ensure the operation of control equipment. In addition, the equipment designer needs to combine the environmental conditions of the equipment to select reliable data for analysis to ensure the accuracy and reliability of the data, and then design an effective solution to make the control equipment safer and more reliable.

\section{Conclusion}

In general, effective research on the reliability of electrical automation control equipment can promote the development of electrical automation technology, and it will greatly help promote the 
socio-economic development of our country. Therefore, the article explores the significance of the reliability research of electrical automation control equipment and proposes relevant development methods to help promote the development of electrical automation control equipment industry.

\section{References}

[1] Chai Li, Ding Xinshan. Research on the reliability of electrical automation-based control equipment [J]. Engineering Technology: Abstracts, 2016 (14): 00277-00277.

[2] Wang Bo. Research on Reliability Testing of Electrical Automation Control Equipment Based on the New Era [J]. Power Technology Applications, 2013 (5).

[3] Li Qingxiang. Research on the reliability of electrical automation control equipment [J]. China Science and Technology Investment, 2017 (22).

[4] Zou Cunzhi. Research on reliability of electrical automation control equipment based on high school students [J]. Electronic Technology and Software Engineering, 2018, No.138 (16): 136.

[5] Jiang Shu Nuan. Reliability Research of Control Equipment Based on Electric Automation [J]. Shandong Industrial Technology, 2016 (22): 246-246.

[6] Chen Guizhou. Research on the Reliability Test of Electrical Automation Control Equipment [J]. Application of Automation, 2018 (04): 75-76. 